\title{
An Analysis of Global Research Trends and Top-Cited Research Articles in Cardio-Oncology
}

\author{
Giselle Alexandra Suero-Abreu $\left.{ }^{\mathrm{a}, \mathrm{c}} \mathbb{(}\right)$, Aldo Barajas-Ochoa ${ }^{\mathrm{a}}{ }^{(0)}$, Robert Berkowitz ${ }^{\mathrm{b}}$
}

\begin{abstract}
Background: As novel cancer therapies continue to improve patient outcomes, there is an increased need for prevention and management of the cardiovascular side effects of these therapies. For this reason, the field of cardio-oncology has experienced significant scientific growth, particularly during the last decade. This study aims to assess the global publication trends and highlight the top-cited scientific articles related to cardio-oncology.
\end{abstract}

Methods: A comprehensive bibliometric analysis of multiple scientific databases was performed to characterize global publication trends in cardio-oncology from 1864 to 2020 and to determine the top-cited papers addressing cardio-oncology as a field of study.

Results: We identified 1,294 publications with 14,494 citations that describe cardio-oncology as a field. Cardio-oncology was the most prevalent term in the literature and was first mentioned in an article from Italy in 1996. There was no further mention of the term "cardiooncology" until 2003, and later again in 2008. After 2010, there was a consistent increase in the number of publications and citations in cardio-oncology. Among the top 50 most cited papers, there was a noticeable trend of higher number of review articles $(\mathrm{n}=28,56 \%$, with 3,208 citations), followed by guidelines and position papers (n $=9,18 \%$, with 2,299 citations) and original research articles $(\mathrm{n}=9$, $18 \%$, with 1,451 citations). The most common specialty for the senior corresponding authors of the top 50 most cited papers was cardiology $(n=36 ; 72 \%)$, followed by oncology $(n=5 ; 10 \%)$; and the most prevalent countries of origin were the USA $(n=26 ; 52 \%)$, Italy $(n=$ $8 ; 16 \%)$, and Canada $(\mathrm{n}=6 ; 12 \%)$.

Conclusion: Our quantitative analysis of publication trends in the field of cardio-oncology objectively showed the growing scientific interest in the field. To our knowledge, this is the first bibliometric

Manuscript submitted June 27, 2021, accepted July 27, 2021

Published online September 9, 2021

aDepartment of Medicine, Rutgers New Jersey Medical School, 150 Bergen Street, Newark, NJ 07103, USA

${ }^{b}$ Heart Failure and Pulmonary Hypertension Program, Hackensack University Medical Center, 30 Prospect Ave, Hackensack, NJ 07601, USA

${ }^{\mathrm{c} C}$ Corresponding Author: Giselle Alexandra Suero-Abreu, Department of Medicine, Rutgers New Jersey Medical School, 150 Bergen Street, Newark, NJ 07103, USA. Email: giselle.suero@gmail.com

doi: https://doi.org/10.14740/cr1291 analysis that determined the top 50 most cited articles in the field of cardio-oncology.

Keywords: Bibliometric analysis; Cardio-oncology; Onco-cardiology; Cardio-hemato-oncology; Research trends; Top articles

\section{Introduction}

Cardio-oncology is an emergent discipline that focuses on preventing and reducing cardiotoxicity related to cancer therapies [1]. Bibliometrics implements statistical methods to analyze publications and explore the growth or impact of a field. It can also provide an objective measure of landmark articles and peer recognition of scientific work by identifying the most cited publications in a given field [2]. As the cardio-oncology field continues to advance, it is important to recognize the significant evolution of this body of academic work and highlight the publications that have introduced and defined the field. In this study, we assessed the global research trends and growth of the field of cardio-oncology and analyzed the top 50 most cited articles.

\section{Materials and Methods}

A bibliometric analysis of the literature in the field of cardiooncology was performed using the multidisciplinary databases Scopus, Pubmed, and Web of Science Core Collection (WOSCC). The terms "cardio-oncology", "onco-cardiology", "cardio-onco-hematology", and "cardio-immuno-oncology" were searched (both hyphenated and non-hyphenated) for all languages for the years 1864 to 2020 . The final search was conducted on March 31, 2021, and the terms were queried as "abstract title, abstract, and keywords" for Scopus and Pubmed, and as "topic" for WOSCC. All articles from the search results were independently verified by two reviewers and were included in the analysis if they addressed cardio-oncology as a field of study in the keywords, title, or abstract. Articles that did not focus on cardio-oncology were excluded. Each article was reviewed for its number of citations, year of publication, and journal of publication. When duplicates were found, the entry with fewer citations was eliminated. For those articles that were co-published in two or more journals, the entry with the highest citations was kept and the citations from the other 
entries were added to this entry. Publication output for each year was analyzed and a list of the 50 most cited articles in the field was generated, as well as a list of journals publishing cardio-oncology articles.

The top 50 most cited articles were reviewed for their citations, year of publication, type of article (i.e., original article, position paper/society guideline, review, systematic review, conference paper, letter to the editor, and case report), journal, and characteristics of the corresponding author (i.e., field of study and country of origin). Due to the public nature of the data used in the study, informed consent for this study was not obtained and it was exempt from Institutional Review Board approval. This study was conducted in compliance with all applicable institutional ethical standards for human study.

\section{Results}

As of March 31, 2021, a total of 1,294 publications related to cardio-oncology as a field with a total of 14,494 citations were found. There was a consistent increase in the number of articles referring to cardio-oncology and the number of citations over the past two decades (Fig. 1). The articles per year were 1 in 1996, 1 in 2003, 2 in 2008, 7 in 2010, 14 in 2011, 12 in 2012, 28 in 2013, 47 in 2014, 75 in 2015, 115 in 2016, 176 in 2017, 184 in 2018, 261 in 2019, and 371 in 2020.

Cardio-oncology was the first term used to describe the field and has remained the most used in the literature compared to the other names associated with the field. In this regard, we identified additional terms referring to the field which arose later in the literature such as: onco-cardiology (in 2008), cardio-onco-hematology (in 2017), and cardio-immuno-oncology (in 2018). The term cardio-oncology was coined in 1996 by Dr. Daniela Cardinale, a cardiologist from Italy, in an article entitled "Una nuova frontiera: la cardioncologia" in the Italian journal Cardiologia (27 citations; first citation in 2010) [3]. There was no further mention of the term "cardio-oncology" until 2003 (one article, one citation), and then later in 2008 (two articles, one and 25 citations). After 2010 and particularly between 2015 and 2020, there has been a significant growth in the number of publications referring to cardio-oncology as a field, likely triggered by the increased recognition of cardiacrelated side effects of novel cancer treatments, such as targeted and immunomodulating therapies.

The top 50 most cited articles in the field of cardio-oncology had a combined total of 7,192 citations as of March 2021 (Table 1). The number of citations per publication ranged from 49 to 986 (mean $143 \pm 157$ ). The most cited articles to date were published between 2010 and 2020, with the majority between 2015 and $2020(\mathrm{n}=31,62 \%)$. All articles were in English and most article types were reviews $(\mathrm{n}=28,56 \%$, with 3,208 citations), followed by guidelines and position papers $(\mathrm{n}=9,18 \%$, with 2,299 citations $)$ and original articles $(\mathrm{n}=9$, $18 \%$, with 1,451 citations). The rest of the articles were one letter to the editor, one systematic review, one case report, and one conference paper.

Among the top 50 most cited papers, the subject matter was varied, with the most common topics being based on clini- cal research or practice. The most cited article was titled "2016 ESC position paper on cancer treatments and cardiovascular toxicity developed under the auspices of the ESC Committee for Practice Guidelines", authored by Zamorano et al and published in 2016 [4]. Interestingly, there were nine guidelines/ position papers among the top 50 most cited articles and three (two by professional societies from cardiology and one from oncology) were among the top five most cited articles [4-6]. The review article "Cardiotoxicity of anticancer drugs: The need for cardio-oncology and cardio-oncological prevention" by Albini et al, published in 2010, was the second most cited paper with 499 citations [1]. The most cited original research article was "Myocarditis in patients treated with immune checkpoint inhibitors" (ICIs) by Mahmood et al (321 citations since its publication in 2018), which highlighted the presentation and clinical course of the potentially fatal myocarditis associated with ICIs [7]. Notably, the most common specialty for the senior corresponding authors of the top 50 most cited papers was cardiology $(n=36 ; 72 \%)$, followed by oncology $(\mathrm{n}=5 ; 10 \%)$. The most prevalent countries of origin were the USA $(n=26)$, Italy $(n=8)$, and Canada $(n=6)$.

Table 2 shows the top 10 journals with the highest number of cardio-oncology publications over time. The list includes two dedicated cardio-oncology journals, JACC Cardio-Oncology and Cardio-Oncology, which launched in 2019 and 2015, respectively. The main focus for the rest of the journals in the list was cardiology. The three journals with the most articles on the 50 most cited list were Journal of the American College of Cardiology (eight publications with 1,347 citations), European Heart Journal (four publications with 1,372 citations), and Circulation (four publications with 241 citations).

\section{Discussion}

This bibliometric analysis of the publication trends in the field of cardio-oncology, from the coining of the term in the first published article in 1996 to date, shows the significant growth of the field in the last years. We quantitatively demonstrated its increasing relevance and attention, based on the number of publications and citations specifically related to the field. In addition, this is the first bibliometric analysis identifying the most cited articles specifically related to cardio-oncology to provide an overview of some of its fundamental developments.

Bibliometric studies of emerging fields and the identification of most cited articles may provide insight on which studies have had the most impact and can help guide further research efforts. However, these assessments may not entirely reflect all the publications that enabled the field's advancement. In this regard, there are many publications of basic, translational, and clinical studies that have contributed significantly to the field of cardio-oncology but have not used the keywords specifically related to the field which were queried in this study. For instance, a paper by Johnson et al published in the New England Journal of Medicine in 2016 highlighted fulminant myocarditis as a serious cardiotoxicity with immune checkpoint blockade, and has more than one thousand citations to date [8]. Nevertheless, this article is not present in the list of 


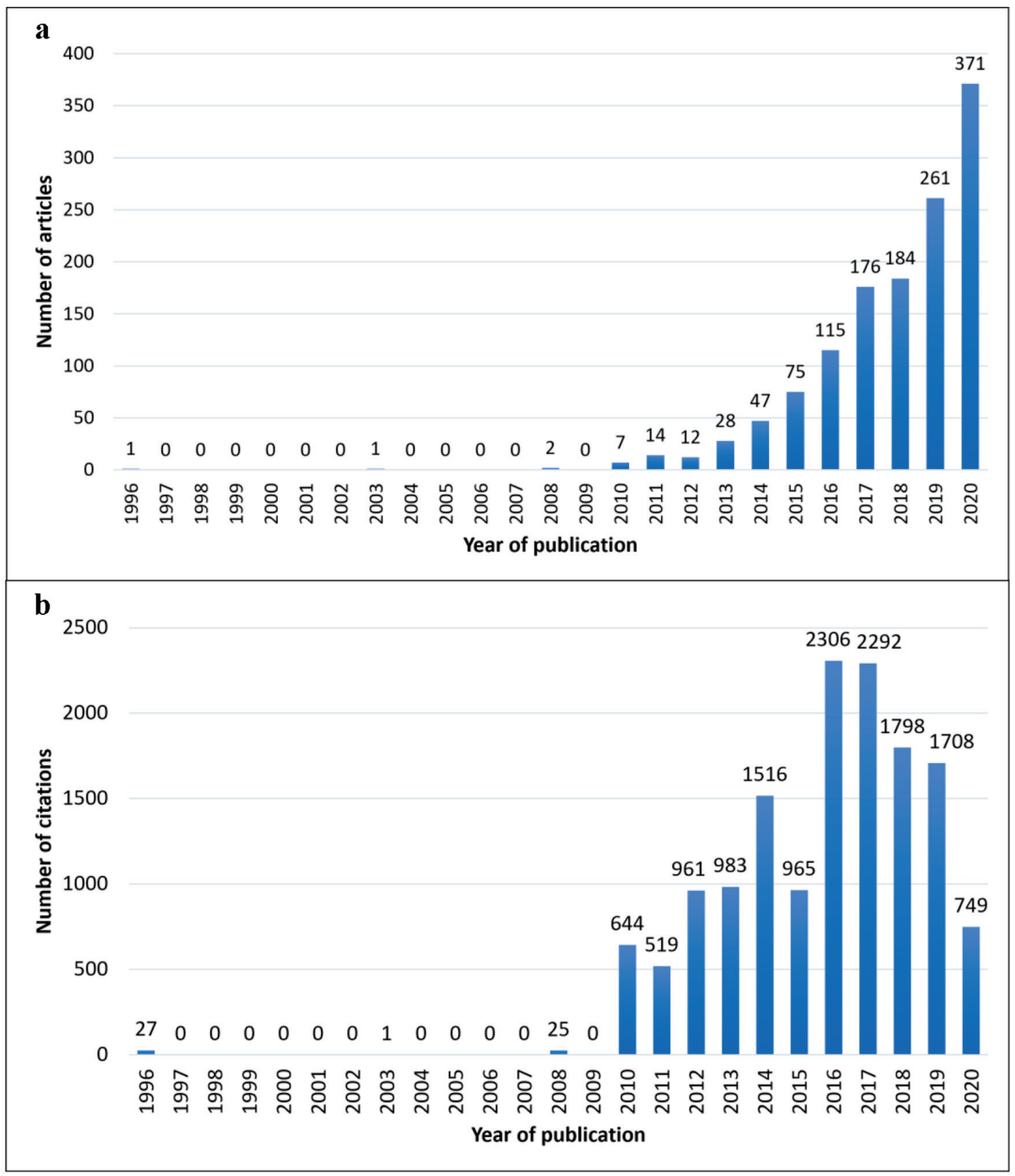

Figure 1. Trends of global cardio-oncology research. Results from bibliometric analysis from 1864 to 2020 illustrate number of publications (a) and number of citations of articles (b) in cardio-oncology.

the top 50 most cited articles because the terms related to the cardio-oncology field per se were not used in the publication title, abstract, or keywords. This highlights the importance of adopting and using standard terms when conducting cardiooncology research to allow for appropriate dissemination of research in the field.

In all the publications analyzed in our study, there was a noticeable trend of higher number of review articles, guidelines/position papers, and letters to the editors compared to original research articles. The main article type among the top
50 articles were reviews which is consistent with the fact that review articles tend to become highly cited in scientific literature [2]. Similarly, nine articles in the top 50 most cited were guidelines/position papers by professional societies and three were among the top five most cited articles. This also reflects the fact that professional society guidelines and position statements usually become widely cited documents within a field, given their reach to a broader audience and direct impact on clinical practice [9]. In general, the main agents associated with cardiotoxicities discussed in the publications included: 


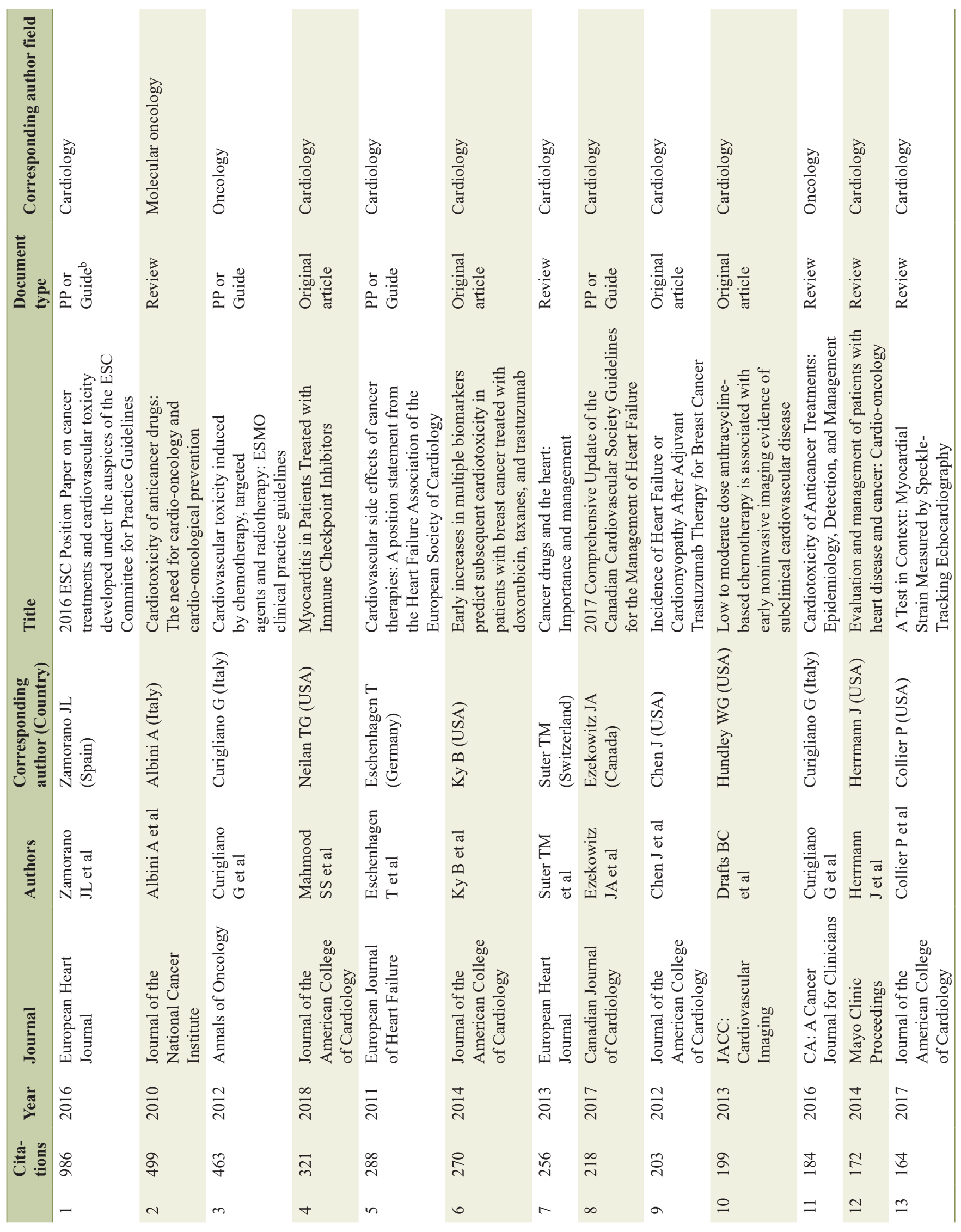




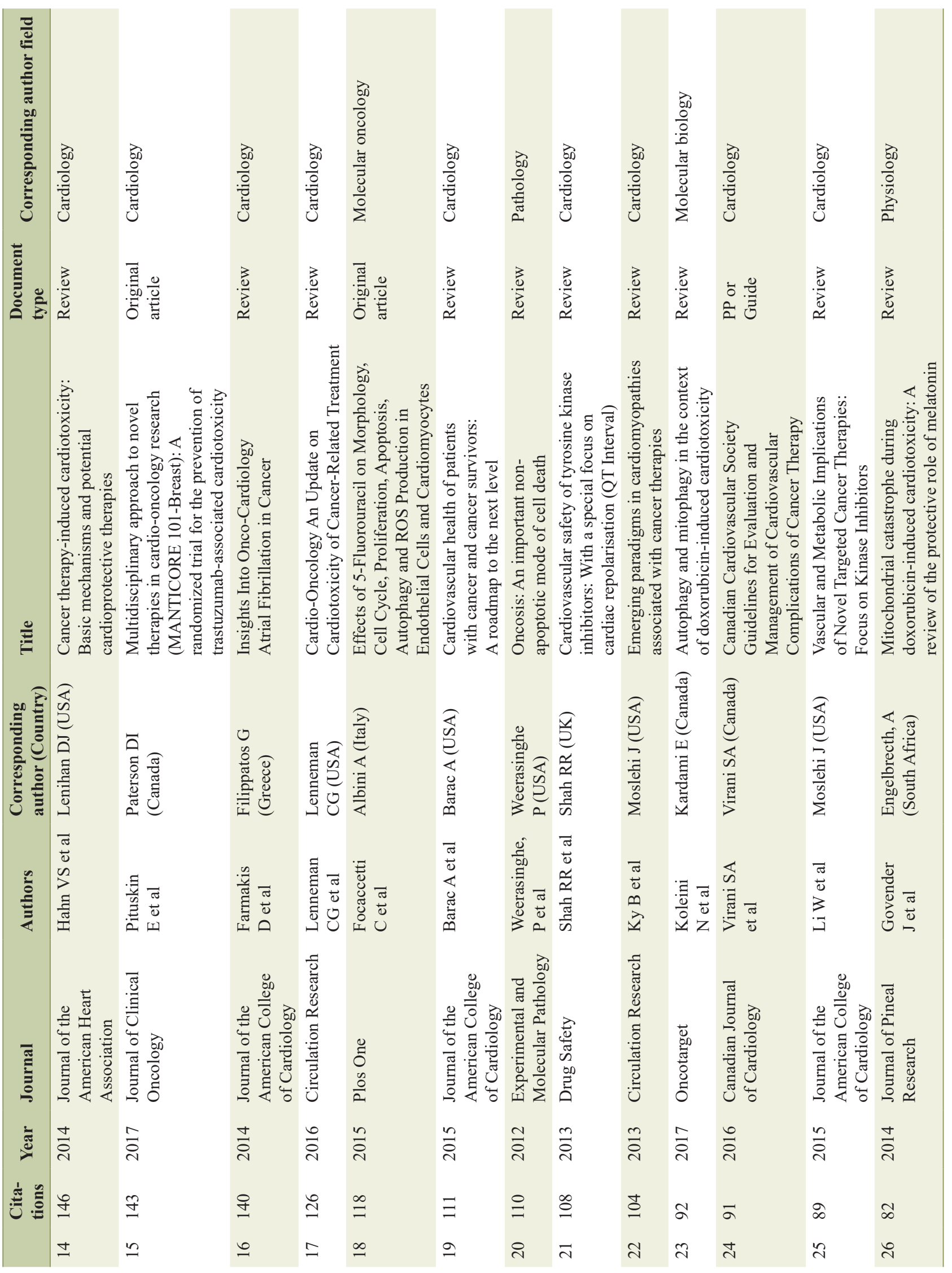



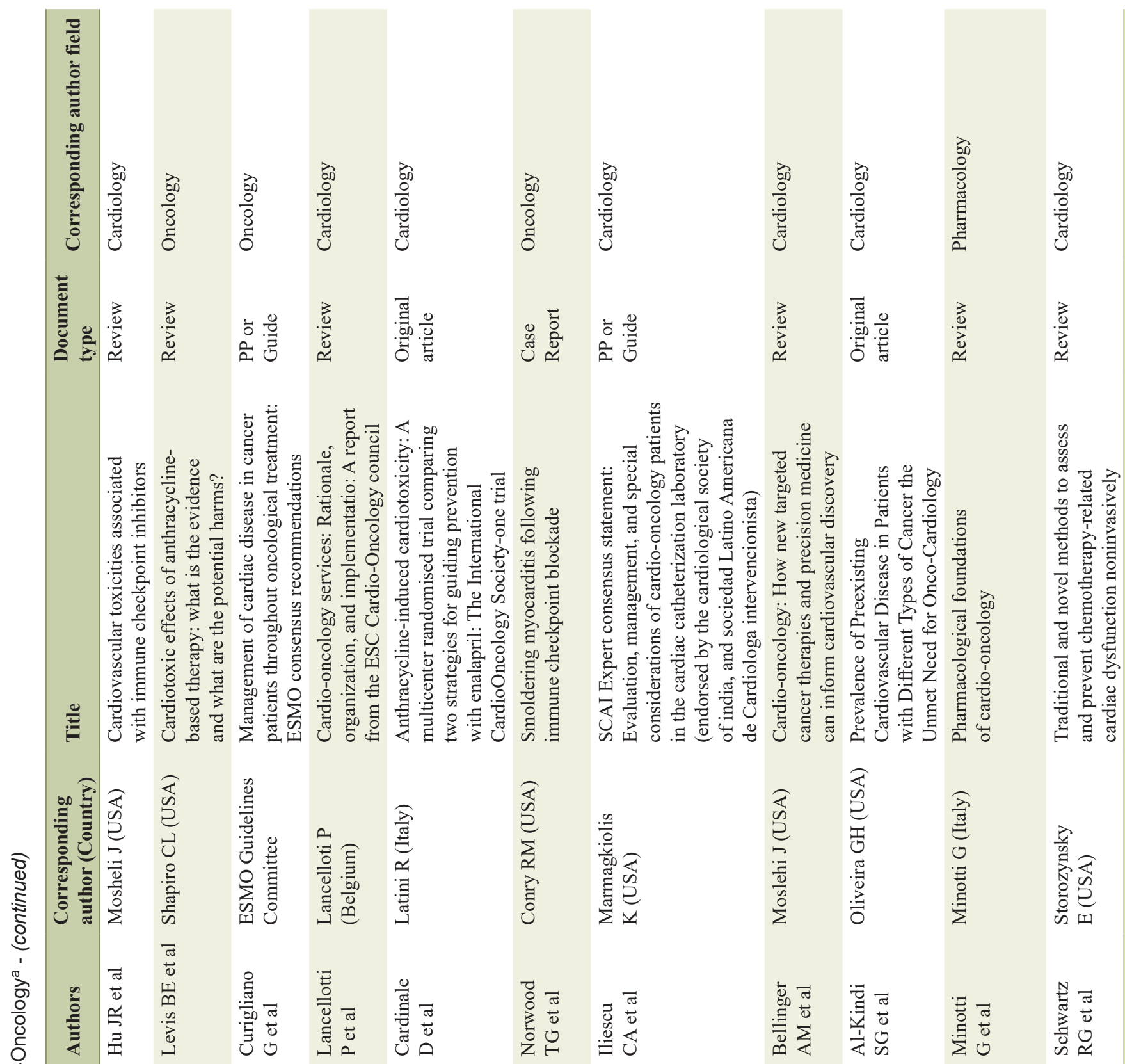

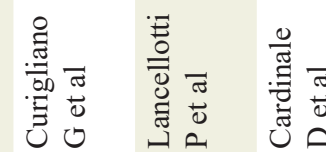

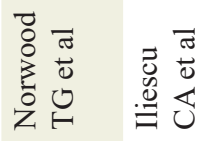

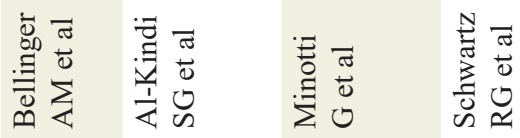
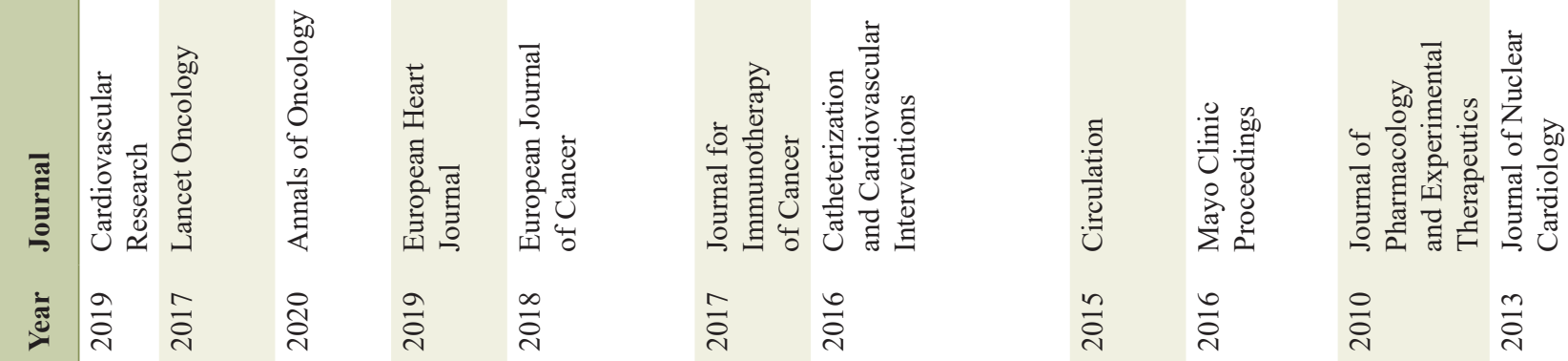

运

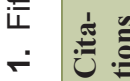

กั๊

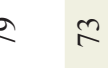

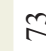

8 8

$\infty \quad \infty$

ลั่ รัก

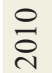

ڤั่

ले m

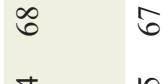

8

3

i $\bar{m}$

m m

只

n 


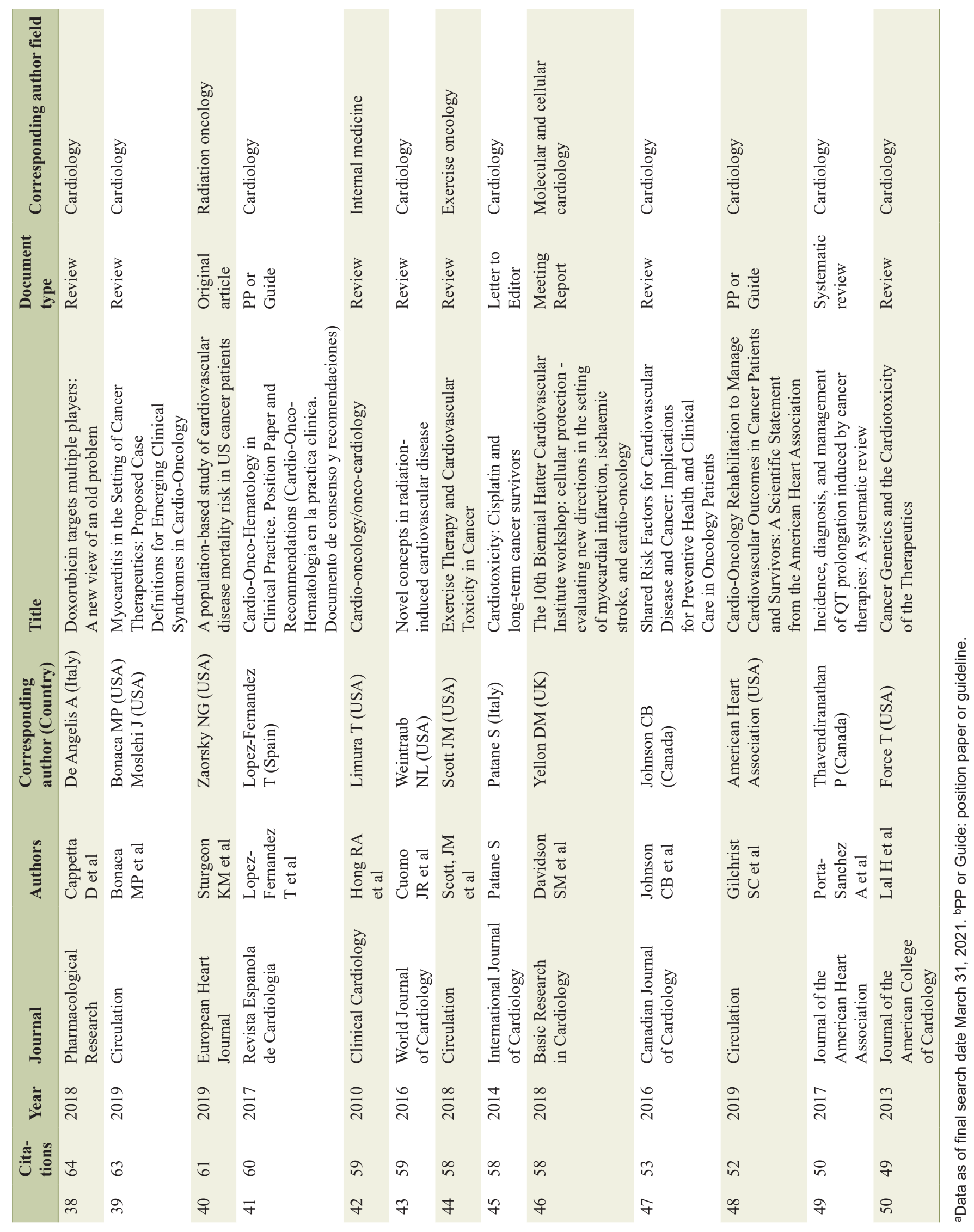


Table 2. Top 10 Journals for Cardio-Oncology Publications ${ }^{a}$

\begin{tabular}{ll}
\hline Journal title & Number of published articles \\
\hline JACC: Cardiooncology & 51 \\
Circulation & 47 \\
International Journal of Cardiology & 40 \\
\hline Journal of the American College of Cardiology & 40 \\
European Journal of Heart Failure & 36 \\
Current Treatment Options in Cardiovascular Medicine & 30 \\
European Heart Journal & 23 \\
Cardiooncology & 22 \\
\hline Journal of the American Heart Association & 19 \\
\hline Current Cardiology Reports & 18 \\
\hline
\end{tabular}

aData as of final search date March 31, 2021.

1) traditional chemotherapy agents such as: antracyclines (i.e., doxorubicin), alkylating agents (i.e., cyclophosphamide), antimetabolites (i.e., 5-fluorouracil, methotrexate, and gemcitabine), and antimicrotubule agents (i.e., paclitaxel, docetaxel, and vincristine); 2) trastuzumab; 3) ICI covering programmed cell death 1 (PD-1) inhibitors (i.e., pembrolizumab and nivolumab) and CTLA-4 inhibitors (i.e., ipilimumab); and 4) tyrosine-kinase inhibitors (i.e., ibrutinib and imatinib).

This bibliometric study used multiple databases and publications in order to capture the growing research trend in cardio-oncology in a comprehensive manner, in contrast with the study of Wei et al where a single database (WOSCC) was queried [10]. Although WOSCC is one of the largest databases for peer-reviewed scientific literature and provides a comprehensive source for bibliometric studies, there are several limitations that need to be considered when querying a single database. Despite a good correlation on the number of indexed articles and citations between several databases, there are known differences among them, leading to articles and citations being missed through the use of a single indexing database [2, 11-14]. In our study, Scopus, WOSCC, and Pubmed were searched in all languages using multiple terms that have been used to refer to the field over the years to avoid missing cardio-oncology publications and citations. This comprehensive approach captured the dynamic nature of this emerging field and resulted in a higher number of articles found in our study when compared to prior publications on the bibliometrics of cardio-oncology. This is illustrated by comparing the 343 publications found by 2018 in studies using a single database [10] with our approach, which found 662 publications by that date.

Although several databases were queried using a comprehensive approach, limitations of this study must be noted. For example, our search strategy was limited to keywords related to cardio-oncology as a field and excluded generic keywords such as "cardiotoxicity" in order to reflect publications that specifically adopted terms that referred to cardio-oncology as a field. As described, this approach did not include some scientific papers related to long-standing research on the cardiotoxic effects of cancer therapies that have created the foundation of knowledge in current cardio-oncology. This can account to the fact that few articles among the top 50 most cited are original research articles. Despite this, our results show that the scientific output specifically classified as cardio-oncology research, including original basic and translational studies, has increased significantly over the past two decades, greatly influenced by cardiology and oncology leaders in the field and supported by dedicated cardio-oncology journals, cardio-oncology societies, and scientific events globally.

\section{Conclusion}

Cardio-oncology has blossomed as a field and its impact is expected to continue to expand. In this study, we showed the growing scientific interest in the field of cardio-oncology and analyzed its top 50 most cited articles. Despite the growth of the field, there is a need to expand our research efforts towards more original basic and translational research studies and to adopt standard terms for the indexing of research related to the field.

\section{Acknowledgments}

None to declare.

\section{Financial Disclosure}

None to declare.

\section{Conflict of Interest}

The authors have no conflict of interest to disclose.

\section{Informed Consent}

Not applicable. 


\section{Author Contributions}

G. A. Suero-Abreu designed the study, engaged in data collection, reviewed the literature, analyzed and interpreted the data, and drafted and reviewed the manuscript. A. Barajas-Ochoa designed the study and search strategy, engaged in data collection, reviewed the literature, analyzed and interpreted the data, and reviewed the manuscript. R. Berkowitz oversaw the design of the study, engaged in data interpretation, and provided critical reviews of the manuscript.

\section{Data Availability}

The authors declare that data supporting the findings of this study are available within the article.

\section{References}

1. Albini A, Pennesi G, Donatelli F, Cammarota R, De Flora S, Noonan DM. Cardiotoxicity of anticancer drugs: the need for cardio-oncology and cardio-oncological prevention. J Natl Cancer Inst. 2010;102(1):14-25.

2. Agarwal A, Durairajanayagam D, Tatagari S, Esteves SC, Harlev A, Henkel R, Roychoudhury S, et al. Bibliometrics: tracking research impact by selecting the appropriate metrics. Asian J Androl. 2016;18(2):296-309.

3. Cardinale D. A new frontier: cardio-oncology. Cardiologia. 1996;41(9):887-891.

4. Zamorano JL, Lancellotti P, Rodriguez Munoz D, Aboyans V, Asteggiano R, Galderisi M, Habib G, et al. 2016 ESC Position Paper on cancer treatments and cardiovascular toxicity developed under the auspices of the ESC Committee for Practice Guidelines: The Task Force for cancer treatments and cardiovascular toxicity of the European Society of Cardiology (ESC). Eur Heart J. 2016;37(36):2768-2801.

5. Curigliano G, Cardinale D, Suter T, Plataniotis G, de
Azambuja E, Sandri MT, Criscitiello C, et al. Cardiovascular toxicity induced by chemotherapy, targeted agents and radiotherapy: ESMO Clinical Practice Guidelines. Ann Oncol. 2012;23(Suppl 7):vii155-166.

6. Eschenhagen T, Force T, Ewer MS, de Keulenaer GW, Suter TM, Anker SD, Avkiran M, et al. Cardiovascular side effects of cancer therapies: a position statement from the Heart Failure Association of the European Society of Cardiology. Eur J Heart Fail. 2011;13(1):1-10.

7. Mahmood SS, Fradley MG, Cohen JV, Nohria A, Reynolds KL, Heinzerling LM, Sullivan RJ, et al. Myocarditis in patients treated with immune checkpoint inhibitors. J Am Coll Cardiol. 2018;71(16):1755-1764.

8. Johnson DB, Balko JM, Compton ML, Chalkias S, Gorham J, Xu Y, Hicks M, et al. Fulminant myocarditis with combination immune checkpoint blockade. N Engl J Med. 2016;375(18):1749-1755.

9. Ioannidis JPA. Professional societies should abstain from authorship of guidelines and disease definition statements. Circ Cardiovasc Qual Outcomes. 2018;11(10):e004889.

10. Wei K, Liao J, Chang J, Zhang X, Chen M, Du J. Bibliometric analysis of the results of cardio-oncology research. Evid Based Complement Alternat Med. 2020;2020:5357917.

11. Archambault E, Campbell D, Gingras Y, Lariviere V. Comparing bibliometric statistics obtained from the Web of Science and Scopus. J Am Soc Inf Sci Technol.. 2009;60(7):1320-1326.

12. Mongeon P, Paul-Hus A. The journal coverage of Web of Science and Scopus: a comparative analysis. Scientometrics. 2016;106(1):213-228.

13. Falagas ME, Pitsouni EI, Malietzis GA, Pappas G. Comparison of PubMed, Scopus, Web of Science, and Google Scholar: strengths and weaknesses. FASEB J. 2008;22(2):338-342.

14. Barajas-Ochoa A, Barajas-Ochoa Z, Ramos-Remus C. Analisis bibliometrico de las revistas medicas del Sistema de Clasificacion de Revistas Mexicanas de Ciencia y Tecnologia. Gac Med Mex. 2019;155(3):258-265. 